\title{
Pathogenic role of angiotensin II and oxidised LDL in obstructive sleep apnoea
}

\author{
T. Kizawa*, Y. Nakamura*, S. Takahashi", S. Sakurai ${ }^{\circ}$, K. Yamauchi* and H. Inoue*
}

ABSTRACT: A sustained elevation of oxidative stress in patients with obstructive sleep apnoea syndrome (OSAS) might help to explain their increased risk for cardiovascular diseases. We tested the hypothesis that the values of oxidative stress are increased in otherwise healthy subjects with OSAS when compared with closely matched control subjects.

We performed a prospective study of 38 subjects who did not have OSAS and 37 patients with OSAS. Plasma indices of angiotensin (Ang) II, vascular endothelial growth factor (VEGF), oxidised low-density lipoprotein (oxLDL), and circulating endothelial precursor cells (CEPs) were measured in OSAS patients and in matched controls. Peripheral blood mononuclear cells (PBMCs) were obtained from both groups and co-cultured with endothelial cells to examine the effects on tube formation.

The OSAS group showed increased levels of Ang II, VEGF, oxLDL and CEPs, which were decreased after nasal continuous positive airway pressure (nCPAP) treatment. In vitro, PBMCs from the OSAS group induced tube formation. Ang II, oxLDL, and Ang II-stimulated PBMCs induced lectin-like oxLDL receptor (LOX-1) expression and VEGF receptor-2 activation on endothelial cells, respectively.

These observations suggest an important role of Ang II and oxLDL-mediated LOX-1 upregulation in endothelial cell injury in patients with OSAS.

KEYWORDS: Angiogenesis, continuous positive airway pressure, obstructive sleep apnoea, oxidant stress, vascular endothelium

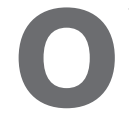
bstructive sleep apnoea syndrome (OSAS) is a highly prevalent disorder affecting $\sim 4 \%$ of adults [1]. The predominant physical morbidity of the disorder is cardiovascular, and OSAS is an independent risk factor for cardiovascular diseases, in particular systemic arterial hypertension [2, 3], but also coronary artery disease, congestive cardiac failure, and cerebrovascular events [4]. In patients with OSAS, oxygen saturation may repeatedly decrease during the apnoeic events. A prominent physiological adaptive response of tissue to hypoxia or ischaemia is angiogenesis, the formation of new blood vessels and increasing the blood supply [5]. Vascular endothelial growth factor (VEGF) regulates multiple endothelial cell (EC) functions including mitogenesis, vascular permeability and vascular tone [6, 7]. VEGF has been shown to be upregulated by angiotensin (Ang) II, which promotes ischaemia-induced angiogenesis in vitro and in vivo [8, 9]. Numerous reports have shown that VEGF expression is significantly increased in the plasma of OSAS patients [10-14] and is induced by Ang II in peripheral blood mononuclear cells (PBMCs) [10]. Furthermore, Ang II upregulates the expression of LOX-1, a lectin-like scavenger receptor for oxidised low-density lipoprotein (oxLDL) in ECs [15]. In turn the activation of LOX-1 also upregulates the Ang II receptor [16]. This positive feedback loop between LOX-1 and Ang II receptor activation increases Ang IIinduced angiogenesis. These angiogenic factors are known to potentiate the proliferation and recruitment of bone-marrow-derived circulating endothelial progenitor cells (CEPs) from bone marrow [15]. Recent studies have provided increasing evidence that the formation of new blood vessels in postnatal life does not result solely from the sprouting of pre-existing vessels, but also involves the recruitment of bonemarrow-derived progenitors for ECs [16]. Postnatal vasculogenesis mainly contributes to vascular healing in response to vascular injury or ischaemia through the processes of rapid endothelialisation of denuded vessels and collateral vessel
AFFILIATIONS

*Division of Pulmonary Medicine, Allergy, and Rheumatology, Dept of Internal Medicine,

\# Dept of Critical Care Medicine, and -Dept of Laboratory Medicine, Iwate Medical University School of Medicine, Morioka, Japan.

\section{CORRESPONDENCE}

Y. Nakamura

Division of Pulmonary Medicine,

Allergy, and Rheumatology, Dept of Internal Medicine

Iwate Medical University School of Medicine

19-1 Uchimaru

Morioka

020-8505 Japan

E-mail: ICB75097@nifty.com

Received:

Jan 202009

Accepted after revision:

May 072009

First published online:

July 022009 
formation [16]. In this process, CEPs home to the site of injury and work in concert with existing mature ECs [16]. It is generally agreed that VEGF is increased in patients with OSAS, but vasculogenesis and the role of CEPs in OSAS is unclear [1719]. Recurrent intermittent hypoxaemia in OSAS may induce the generation of an excess of reactive oxygen species (ROS), which can ultimately result in endothelial cellular injury via reactions with proteins, nucleic acids and lipids. Whether there is indeed increased oxidative stress in OSAS is controversial [20-24]. These conflicting results may be explained by several factors including the presence of comorbidities and medications in OSAS patients, both of which can have significant effects on measurements of oxidative stress. OSAS may be a hallmark of CEPs' mobilisation and oxidative stress.

To more easily recognise the impact of these factors, more research must be done so that their effects and the roles that they play in obtaining results can be better understood. Therefore it was the purpose of this study to test the hypothesis that the plasma level of angiogenic factors, CEPs and the lipid peroxidation are increased in otherwise healthy subjects with OSAS when compared with closely matched control subjects proved to be free of any other diseases. By proving this hypothesis, we can show a part of the mechanism that underlies OSAS that will help us to better understand the many factors that contribute to this ailment.

\section{MATERIALS AND METHODS}

\section{Patients and control subjects}

Males with suspected OSAS, who had sleep-related symptoms (i.e. snoring, witnessed apnoeas and excessive daytime sleepiness) and no other medical disorders, were considered for the study, which was approved by the Iwate Medical University Hospital Ethics Committee from the patients who sought treatment within their facilities. Prospective patients were then notified of our desire to include them in our study and asked if they would be willing to participate. Upon acceptance, the subjects were provided written informed consent according to the ethical protocols of our institution (see online supplementary data).

\section{Measurement of Ang II, VEGF and oxLDL}

Blood samples were drawn from subjects' antecubital veins at the end of each sleep study (i.e. 6:00AM). Further procedures are given in the online supplementary data.

\section{Quantification of CEPs}

We studied the peripheral blood level of CEPs in eight of the most severe OSAS patients whose apnoea/hypopnoea index (AHI) values were $>60$, and eight age- and body mass index (BMI)matched control subjects (see online supplementary data).

\section{Reverse transcriptase PCR for mRNA expression and immunoblotting analysis of LOX-1 on human aortic ECs}

Human aortic ECs (HAECs) were purchased from Kurabo (Osaka, Japan) and maintained in a Humedia-EG2 medium (Kurabo) according to the manufacturer's instructions. Stimulated HAECs were submitted to reverse transcriptase (RT)-PCR and immunoblotting [25] (also see the online supplementary data).

\section{For co-culture of PBMCs treatment with human umbilical vein ECs or HAECs}

In the co-culture test, we used PBMCs obtained from eight severe OSAS patients and eight age- and BMI-matched control subjects. Heparinised venous blood was centrifuged on Histopaque 1077 (Sigma, Tokyo, Japan) at 600xg for $20 \mathrm{~min}$ at room temperature. The detailed protocol of PBMCs' isolation has been reported elsewhere [10] (see online supplementary data). Co-culture experiments were performed in 24-well plate ThinCert Cell Culture Inserts systems (membranes with pore size of $0.4 \mu \mathrm{m}$ in diameter, growth area of membrane $33.6 \mathrm{~mm}^{2}$, Greiner Bio-One, Frickenhausen, Germany) with HAECs or human vein ECs (HUVECs) plated at the bottom of the wells. PBMCs $\left(1 \times 10^{6} \cdot \mathrm{mL}^{-1}\right.$ or $\left.1 \times 10^{4} \cdot \mathrm{mL}^{-1}\right)$ were seeded onto the inserts and thereafter incubated overnight at $37^{\circ} \mathrm{C}, 5 \% \mathrm{CO}_{2}$ in air for the indicated times.

\section{Modified in-vitro co-culture angiogenesis}

In vitro angiogenesis was assessed by the formation of capillary-like structures of HUVECs co-cultured with human

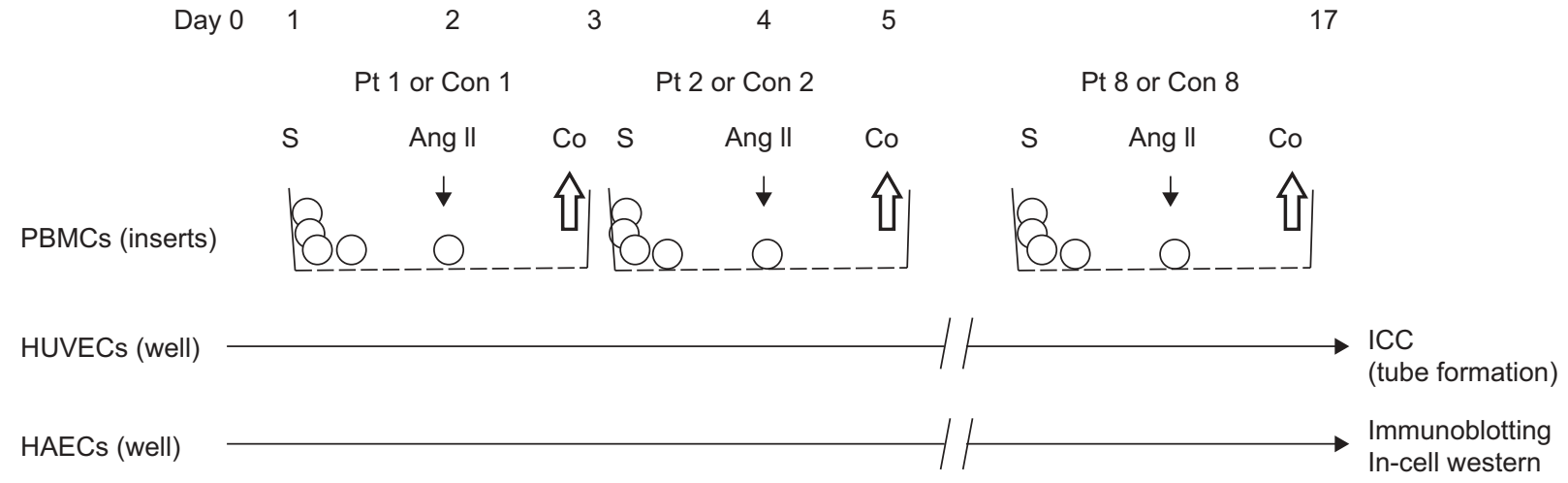

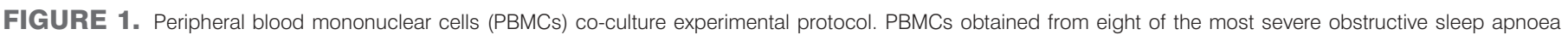

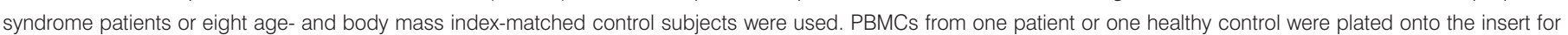

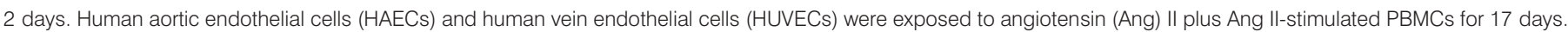

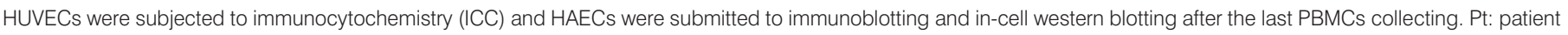
with obstructive sleep apnoea; Con: healthy control subject; S: seeding and starvation; Co: collection of PBMCs and medium. 


\begin{tabular}{|c|c|c|c|c|}
\hline \multirow[t]{2}{*}{ TABLE 1} & \multicolumn{4}{|c|}{$\begin{array}{l}\text { Demographic, sleep and clinical data of control } \\
\text { subjects and obstructive sleep apnoea } \\
\text { syndrome (OSAS) patients }\end{array}$} \\
\hline & & Control & OSAS & p-value \\
\hline \multicolumn{2}{|l|}{ Subjects $n$} & 38 & 37 & \\
\hline \multicolumn{2}{|c|}{ Age yrs } & $45.2 \pm 2.3$ & $46.4 \pm 2.6$ & 0.7 \\
\hline \multicolumn{2}{|c|}{ Body mass index $\mathrm{kg} \cdot \mathrm{m}^{-2}$} & $30.0 \pm 1.0$ & $28.8 \pm 1.0$ & 0.4 \\
\hline \multicolumn{5}{|c|}{ Full blood count } \\
\hline \multicolumn{2}{|c|}{$\mathrm{WBC} \cdot \mu \mathrm{L}^{-1}$} & $5926.3 \pm 304.0$ & $6167.6 \pm 329.2$ & 0.6 \\
\hline \multicolumn{2}{|l|}{$\mathrm{Hb} g \cdot \mathrm{dL}^{-1}$} & $14.5 \pm 0.2$ & $14.9 \pm 0.3$ & 0.3 \\
\hline \multicolumn{2}{|c|}{ PLT $\times 10^{4} \cdot \mu L^{-1}$} & $26.0 \pm 1.4$ & $29.0 \pm 1.3$ & 0.1 \\
\hline \multicolumn{5}{|c|}{ Fasting lipids $\mathrm{mg} \cdot \mathrm{dL}^{-1}$} \\
\hline \multicolumn{2}{|c|}{ Total cholesterol } & $177.0 \pm 5.0$ & $185.0 \pm 4.1$ & 0.2 \\
\hline \multicolumn{2}{|c|}{ Triglycerides } & $105.0 \pm 3.4$ & $101.0 \pm 3.6$ & 0.4 \\
\hline \multicolumn{2}{|c|}{ HDL cholesterol } & $66.1 \pm 1.8$ & $64.8 \pm 1.9$ & 0.6 \\
\hline \multicolumn{2}{|c|}{ LDL cholesterol } & $89.8 \pm 5.3$ & $99.9 \pm 4.1$ & 0.1 \\
\hline \multicolumn{2}{|c|}{ Fasting plasma glucose $\mathrm{mg} \cdot \mathrm{dL}^{-1}$} & $98.2 \pm 1.2$ & $95.6 \pm 1.4$ & 0.2 \\
\hline \multicolumn{2}{|c|}{$\mathrm{Hb}$ A1c \% } & $4.8 \pm 0.1$ & $4.9 \pm 0.1$ & 0.3 \\
\hline \multicolumn{2}{|l|}{ AST IU $\cdot \Gamma^{-1}$} & $28.8 \pm 1.4$ & $27.1 \pm 1.4$ & 0.4 \\
\hline \multicolumn{2}{|c|}{ ALT IU $\cdot I^{-1}$} & $23.7 \pm 1.2$ & $25.5 \pm 1.6$ & 0.4 \\
\hline \multicolumn{2}{|c|}{ Systolic blood pressure $\mathrm{mmHg}$} & $123.7 \pm 1.9$ & $125.3 \pm 2.0$ & 0.6 \\
\hline \multicolumn{2}{|c|}{ Diastolic blood pressure $\mathrm{mmHg}$} & $72.3 \pm 1.6$ & $74.3 \pm 1.7$ & 0.4 \\
\hline \multicolumn{5}{|c|}{ Sleep study details } \\
\hline \multicolumn{2}{|c|}{$\mathrm{AHI}$ events $\cdot \mathrm{h}^{-1}$ of sleep } & $2.1 \pm 0.2$ & $37.2 \pm 3.2$ & $<0.001$ \\
\hline \multicolumn{2}{|c|}{ Average nightly $\mathrm{SaO}_{2} \%$} & $93.9 \pm 0.5$ & $83.3 \pm 0.9$ & $<0.001$ \\
\hline \multicolumn{2}{|c|}{ Minimal $\mathrm{SaO}_{2} \%$} & $88.3 \pm 0.6$ & $68.4 \pm 1.3$ & $<0.001$ \\
\hline \multicolumn{2}{|c|}{$\%$ total sleep time $<\mathrm{SaO}_{2} 90 \% \%$} & $2.0 \pm 0.3$ & $15.7 \pm 1.1$ & $<0.001$ \\
\hline
\end{tabular}

Data are expressed as mean \pm SEM, unless otherwise stated. WBC: white blood cell; Hb: haemoglobin; PLT: platelet; HDL: high-density lipoprotein; LDL: lowdensity lipoprotein; AST: aspartate aminotransferase; ALT: alanine aminotransferase; $\mathrm{AHI}$ : apnoea/hypopnoea index; $\mathrm{SaO}_{2}$ : oxygen saturation. Bold p-values are statistically significant.

diploid fibroblasts (see online supplementary data). The experimental procedure followed the instructions provided with the Angiogenesis kit (Kurabo). On day 3, PBMCs were collected and RNA was extracted from PBMCs (see online supplementary data). The new PBMCs from another patient were seeded onto the inserts in the new medium, starved for $24 \mathrm{~h}$, and then followed by repeated stimulation and preincubation. This procedure was repeated on days 5, 7, 9, 11, 13 and 15 . On day 17 HUVECs were submitted to counter staining (fig. 1, also see online supplementary material).

\section{Co-culture for LOX-1 expression and VEGF receptor-2 phosphorylation on HAECs}

HAECs $\left(1 \times 10^{5}\right)$ were plated $72 \mathrm{~h}$ prior to assay in 24-well plates so they would be 50 to $60 \%$ confluent when co-cultured with PBMCs, which were seeded onto inserts on day 1 . The replacement of PBMCs in the inserts and medium, and the condition of incubation with Ang II, valsartan, or soluble VEGF receptor (VEGFR)-2, were as described in the procedure of in vitro angiogenesis. On day 17, HAECs were submitted to immunoblotting to detect LOX-1 protein expression (see online supplementary data) or to examine VEGFR-2 phosphorylation using the in-cell western blotting method [26] (fig. 1, also see online supplementary data).

\begin{tabular}{|c|c|c|c|c|}
\hline \multirow[t]{2}{*}{ TABLE 2} & \multicolumn{4}{|c|}{$\begin{array}{l}\text { Specific laboratory data for oxidised low-density } \\
\text { lipoprotein (oxLDL) and \% circulating endothelial } \\
\text { progenitor cells (CEPS) }\end{array}$} \\
\hline & & Control & OSAS & Post-CPAP \\
\hline \multicolumn{2}{|c|}{ Plasma oxLDL U. $\mathrm{L}^{-1}$} & $32.3 \pm 2.7$ & $43.6 \pm 3.6^{*}$ & $32.7 \pm 3.2$ \\
\hline \multicolumn{2}{|c|}{$\begin{array}{l}\% \text { CEPs (of white blood } \\
\text { cell number) }\end{array}$} & $0.48 \pm 0.09$ & $1.40 \pm 0.33^{*}$ & $0.75 \pm 0.18$ \\
\hline
\end{tabular}

Data are presented as mean \pm SEM. OSAS: obstructive sleep apnoea syndrome; CPAP: continuous positive airway pressure. ${ }^{*}: p<0.05$ versus control and post-CPAP

\section{Statistical analysis}

All data are expressed as the mean \pm SEM. The one-way ANOVA and post hoc tests were used to assess the statistical significance among the groups. An unpaired t-test was also used to examine changes between the control group and the OSAS patients after testing data for the normality of the population using the Kolmogorov-Smirnov test.

\section{RESULTS}

\section{Patient and control population}

Of the 77 subjects initially examined, 39 patients had OSAS. Since two of the subjects were newly diagnosed with asthma and allergic rhinitis after the study, the non-OSAS control and the OSAS group consisted of 38 and 37 individuals, respectively. The non-OSAS control individuals were informed of the clinical features of OSAS, including significant excessive daytime sleepiness and snoring, but demonstrated no objective finding of sleep-disordered breathing in polysomnography (PSG) studies. The baseline characteristics of the study population are described in table 1. The two groups were similar in age and moderate obesity. AHI and oximetry data were significantly different between the two groups $(p<0.01)$. No subject had clinical evidence of any other medical disorder, and each subject's detailed biochemical profile, including liver and renal function, cardiac enzymes and resting electrocardiogram, were within normal limits. Of the original 37 OSAS patients recruited, only the eight severe OSAS patients were used for this in vitro study because they had an AHI $>60$, control patients with similar age and BMI were used.

\section{Measures of Ang II, VEGF and oxLDL}

Table 2 shows the levels of oxLDL in the present study. In total, 29 suitable subjects with OSAS, who had agreed to possible continuous positive airway pressure (CPAP) therapy before undergoing the diagnostic sleep study, were considered. Eight subjects dropped out due to intolerance of CPAP. The remaining 29 subjects underwent repeated sleep studies after 12 weeks of therapy. All were evaluated for symptoms and side-effects, and objective compliance data was downloaded from the devices. In patients with OSAS, the levels of Ang II, VEGF and oxLDL were significantly higher than in the control and post-CPAP group (table 2; Ang II and VEGF data not shown). After 12 weeks of CPAP therapy in the 29 CPAP-treated subjects with OSAS, all three data points decreased (table 2), but there was no noticeable decrease in other observed parameters. 
a)
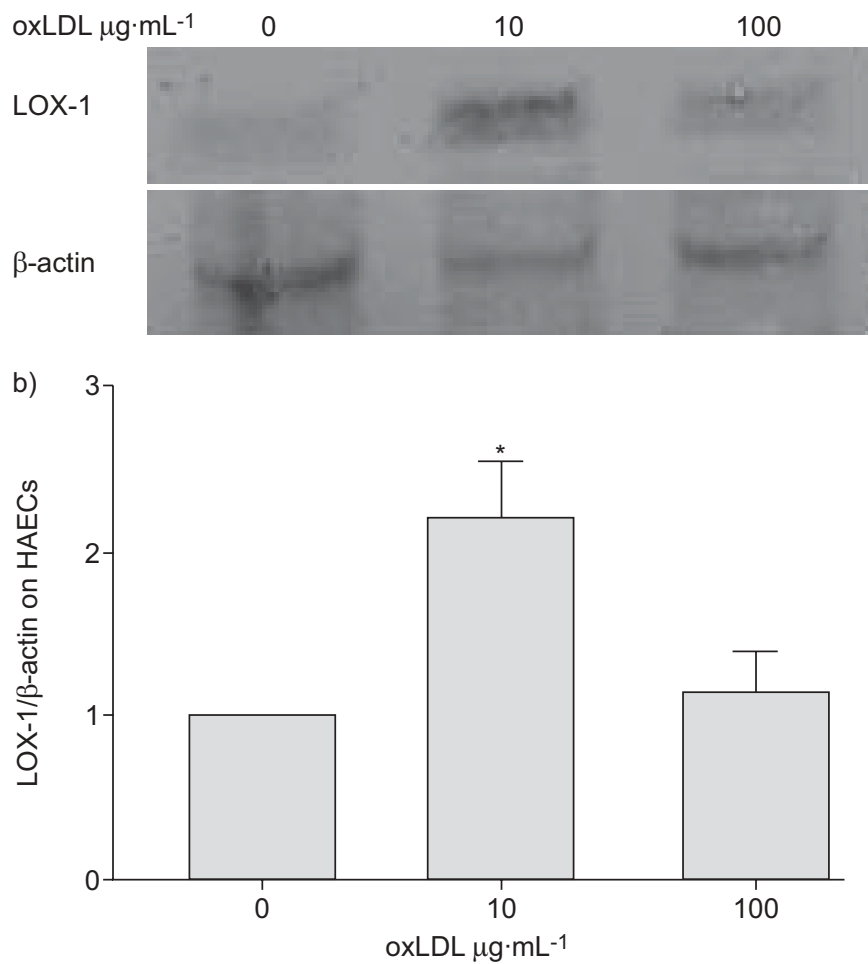

FIGURE 2. Endothelial receptor for lectin-like oxidised low-density lipoprotein (oxLDL) on human aortic endothelial cells (HAECs). The incubation of HAECs with oxLDL for 3 days upregulated the expression of lectin-like oxLDL receptor (LOX-1) mRNA. oxLDL at a concentration of $10 \mu \mathrm{g} \cdot \mathrm{mL}^{-1}$ increased LOX-1 mRNA expression on HAECs. In contrast, $100 \mu \mathrm{g} \cdot \mathrm{mL}^{-1}$ of oxLDL induced less expression. LOX-1 protein band density was normalised by the housekeeping gene $\beta$-actin a) Representative of five separate experiments. b) Summary of the data (mean \pm SEM) from five experiments. ${ }^{*}: \mathrm{p}<0.05$ versus $0 \mu \mathrm{g} \cdot \mathrm{mL}^{-1}$.

\section{The numbers of bone-marrow-derived CEPs}

Hypoxia in the night and high concentrations of Ang II and VEGF that were found in the circulation of patients with OSAS strongly suggested that CEPs and their stem cells in the bone marrow responded to these angiogenic factors. White blood cells from eight of the most severe OSAS patients (AHI >60) and controls were used for this experiment and the subsequent experiments of co-cultivation with ECs. Flow-cytometric analyses for the staining of CD133, CD34, and CD202b (Tie-2) on the gated CD45-negative cells of the participants are shown in table 2 . Individuals with OSAS had a $\sim 3$-fold increase in $\mathrm{CD} 133^{+} \mathrm{CD} 34^{+} \mathrm{CD} 202 \mathrm{~b}^{+} \mathrm{CD} 45^{-}$cells in their blood circulation compared with the control group. After CPAP treatment, this increase was suppressed (table 2).

\section{Influence of Ang II and oxLDL on LOX-1 expression in HAECs}

Having shown the increased plasma levels of Ang II, VEGF and oxLDL in the OSAS patients, we next investigated the interaction between these molecules and HAECs in vitro. We evaluated whether oxLDL and Ang II induce LOX-1 expression on HAECs.

As shown in figure 2, a low concentration of oxLDL led to the expression of LOX-1. The maximum LOX-1 expression occurred a)
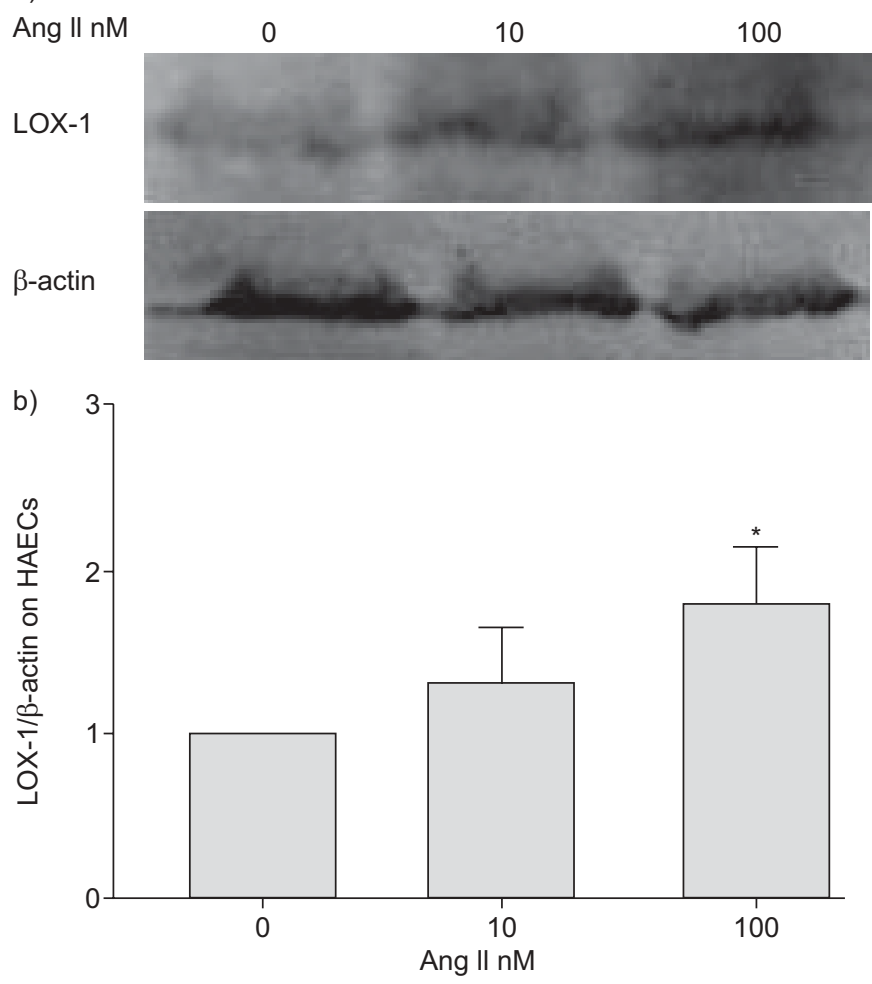

FIGURE 3. Lectin-like oxidised low-density lipoprotein (oxLDL) receptor (LOX-1) protein expression on human aortic endothelial cells (HAECs). Incubation of HAECs with angiotensin (Ang) II for 3 days increased the expression of LOX-1 mRNA in a concentration-dependent manner. a) Representative of five separate experiments. b) Summary of the data (mean \pm SEM) from five experiments. *: $\mathrm{p}<0.05$ versus $0 \mathrm{nM}$.

in response to a $10 \mu \mathrm{g} \cdot \mathrm{mL}^{-1}$ concentration of oxLDL (fig. 2). In contrast, we observed that LOX-1 expression increased in response to Ang II in a concentration-dependent fashion (fig. 3). Based on these findings, we chose the maximum effective concentrations of oxLDL $\left(10 \mu \mathrm{g} \cdot \mathrm{mL}^{-1}\right)$ and Ang II $(100 \mathrm{nM})$ for the subsequent experiments. When HAECs were stimulated with oxLDL-1 and Ang II, the level (mRNA and protein) of LOX-1 was additive for both stimuli (fig. 4).

\section{PBMCs from OSAS patients induce tube formation via VEGFR-2 phosphorylation in the modified co-culture system}

The increase of LOX-1 expression by Ang II led us to determine whether this molecule affects another known response of ECs, angiogenesis. Since Ang II has been reported to be able to induce VEGF from PBMCs [10], we thought it possible that the tube formation is dependent on VEGF which may be secreted from PBMCs. To test this idea, we put inserts in the angiogenesis kit and seeded PBMCs onto the inserts to detect their interaction with HUVECs (fig. 1). As shown in figure $5 a$ and $b$, the tube formation by co-cultivation with PBMCs was significantly increased when the PBMCs were exposed to Ang II in vivo and were obtained from OSAS patients whereas little tube formation was observed when the cells were exposed to medium only. This effect of PBMCs obtained from OSAS patients was absent in the presence of soluble VEGFR (sVEGFR)-2. Similar results in the mRNA level of VEGF165 

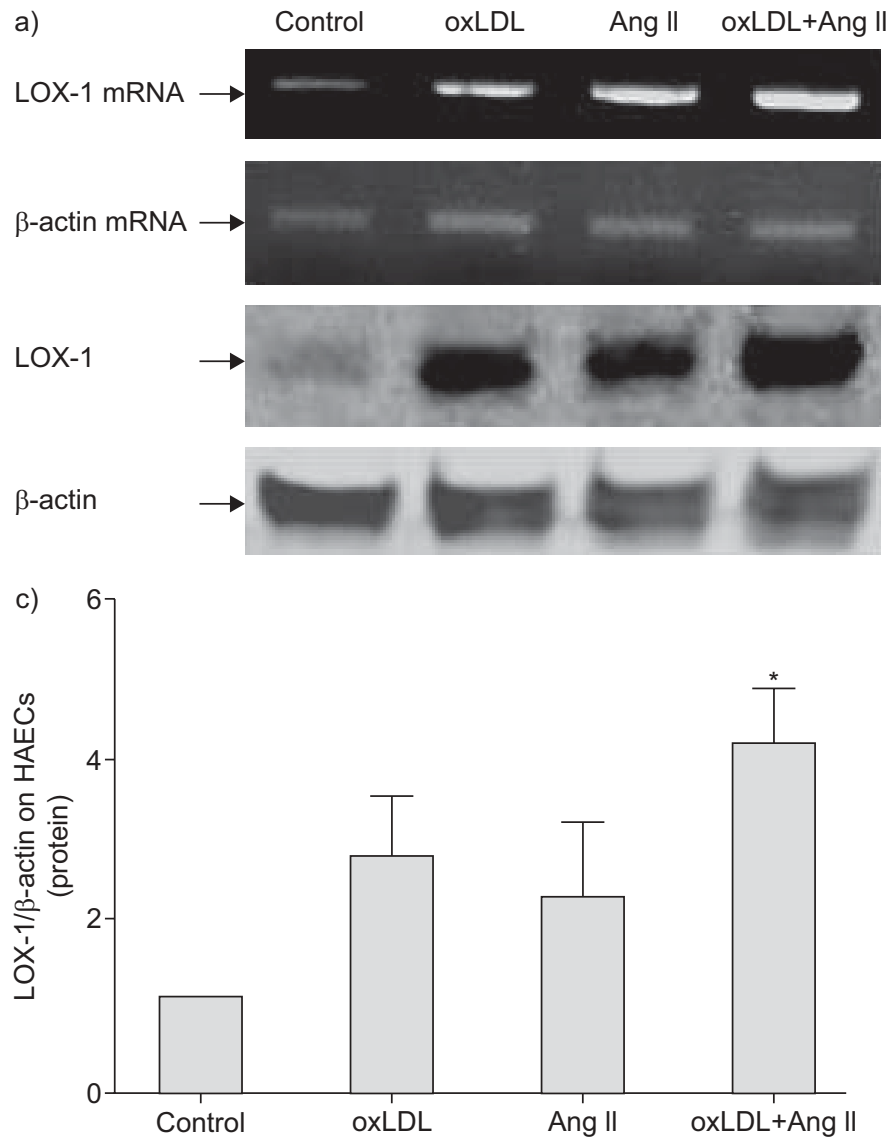

were observed when PBMCs were subjected to RT-PCR (data not shown). Of note, sVEGFR-2 did not interfere with VEGF165 mRNA expression in PBMCs. These results suggest that VEGF from OSAS PBMCs contributes to tube formation. As PBMCs from patients with OSAS expressed VEGF, cultured PBMCs from normal subjects were further analysed in tubular formation and phosphorylation of ECs experiments after treatment with Ang II. As shown in figure 6a and b, the tube formation by PBMCs from controls was significantly increased after stimulation with Ang II. The biological effects of Ang II are mediated by the activation of Ang II receptors, of which two major subtypes, AT1R and AT2R, have been identified. Most of the cardiovascular actions of Ang II have been attributed to AT1R activation. Pre-treatment with AT1R blocker valsartan and sVEGFR-2 markedly suppressed the tube formation induced by PBMCs from control subjects with Ang II stimulation. The inhibition of tube formation by these factors was not due to any expected toxicity, because nonspecific immunoglobulin (Ig) G could not inhibit tubular formation. These results clearly indicated that the tube formation induced by PBMCs from controls was highly dependent on VEGF secretion. Having identified Ang II induced-VEGF in PBMCs as an inducer of EC tube formation, we next sought to confirm the activation of VEGFR-2 on HAECs. It has previously been reported that tyrosine residues on VEGFR-2 are autophosphorylated in response to VEGF, and that the phosphorylation of VEGFR-2 at Tyr ${ }^{1214}$ has a central role in the activation of this receptor. PBMCs from OSAS led to the dose-dependent phosphorylation of VEGFR-2 at $\mathrm{Tyr}^{1214}$ $(\mathrm{p}<0.05$; fig. $5 \mathrm{a}$ and $\mathrm{c})$. sVEGFR-2 strongly inhibited the

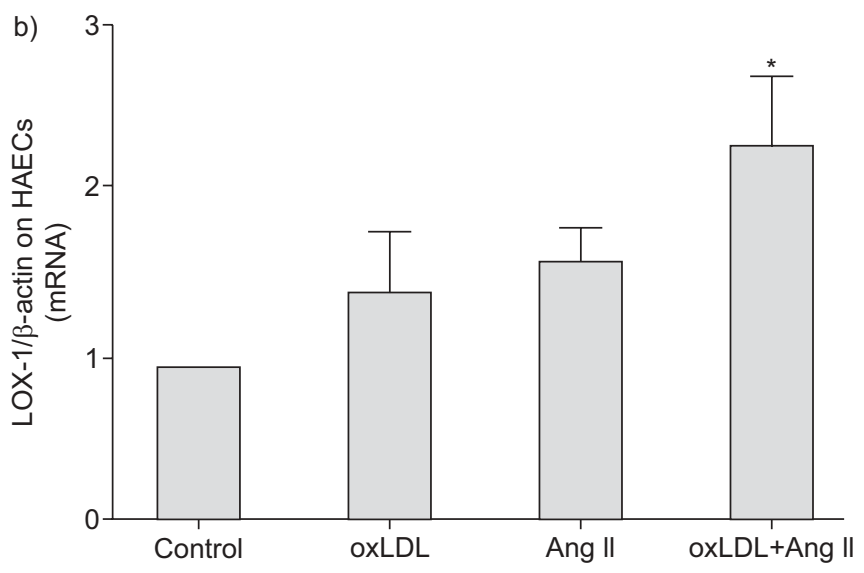

FIGURE 4. Upregulation of lectin-like oxidised low-density lipoprotein (oxLDL) receptor (LOX-1) mRNA and protein expression by oxLDL and angiotensin (Ang) ॥ in human aortic endothelial cells (HAECs). Treatment with either oxLDL or Ang ॥ caused a modest increase in LOX-1 expression in HAECs. The presence of both oxLDL and Ang II increased LOX-1 expression in a cumulative fashion. a) Representative of five separate experiments. b and c) Summary of data (mean \pm SEM) from 5 experiments. ${ }^{*}: p<0.05$ versus control.

stimulatory effect of the phosphorylation of VEGFR-2 on HAECs (fig. 5a and c). In keeping with our tubular formation data, the increased receptor phosphorylation occurred in response to co-cultivation with Ang II-stimulated PBMCs or VEGF stimulation (fig. $6 a$ and $c ; p<0.05$ ). Pre-treatment with valsartan and sVEGFR-2, but not nonspecific IgG, markedly suppressed the VEGFR-2 phosphorylation by Ang II- stimulated PBMCs. These increased tubular- formed ECs expressed LOX-1 protein (fig. 6a). Thus, we concluded that Ang II induced VEGF via the AT1R on PBMCs, resulting in an increase in tube formation and the expression of LOX-1 protein.

\section{DISCUSSION}

Several new findings have emanated from this study. First, we have demonstrated enhanced angiogenesis, vasculogenesis and lipid peroxidation, oxLDL in OSAS patients when compared with controls, which was reversed by CPAP therapy. Secondly, we have shown that oxLDL and Ang II induced LOX-1 expression on ECs. Finally, we have shown that Ang II induced EC tube formation, which express LOX-1, via PBMCs.

Our in vivo study has a number of strengths compared with some previous studies. We took care in selecting subjects to exclude smokers, anyone with cardiovascular or other medical disorders, or who was taking any medication. Furthermore, we excluded subjects with sunburn, because even physiological doses of ultraviolet $\mathrm{A}$ radiation administered at above $300 \mathrm{~W} \cdot \mathrm{m}^{-2}$ have previously been shown to induce oxidative 
a) PBMC

$\frac{\text { Control }}{1 \times 10^{6}} \frac{\text { OSAS }}{1 \times 10^{4}}+1 \times 10^{6}+$ +sVEGFR-2

Tube formation

EC
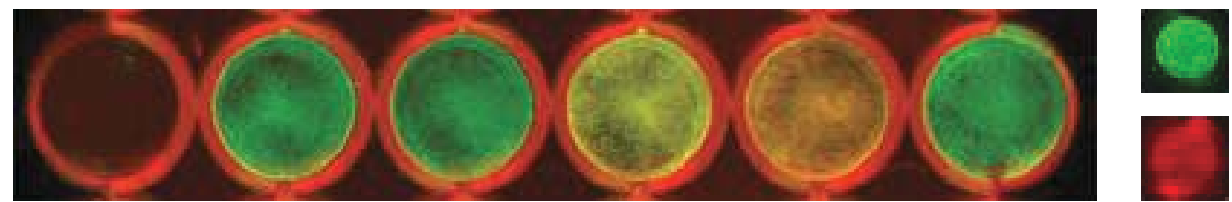

$800 \mathrm{~nm}$ image: VEGFR-2

$700 \mathrm{~nm}$ image:

Phosphorylated VEGFR-2
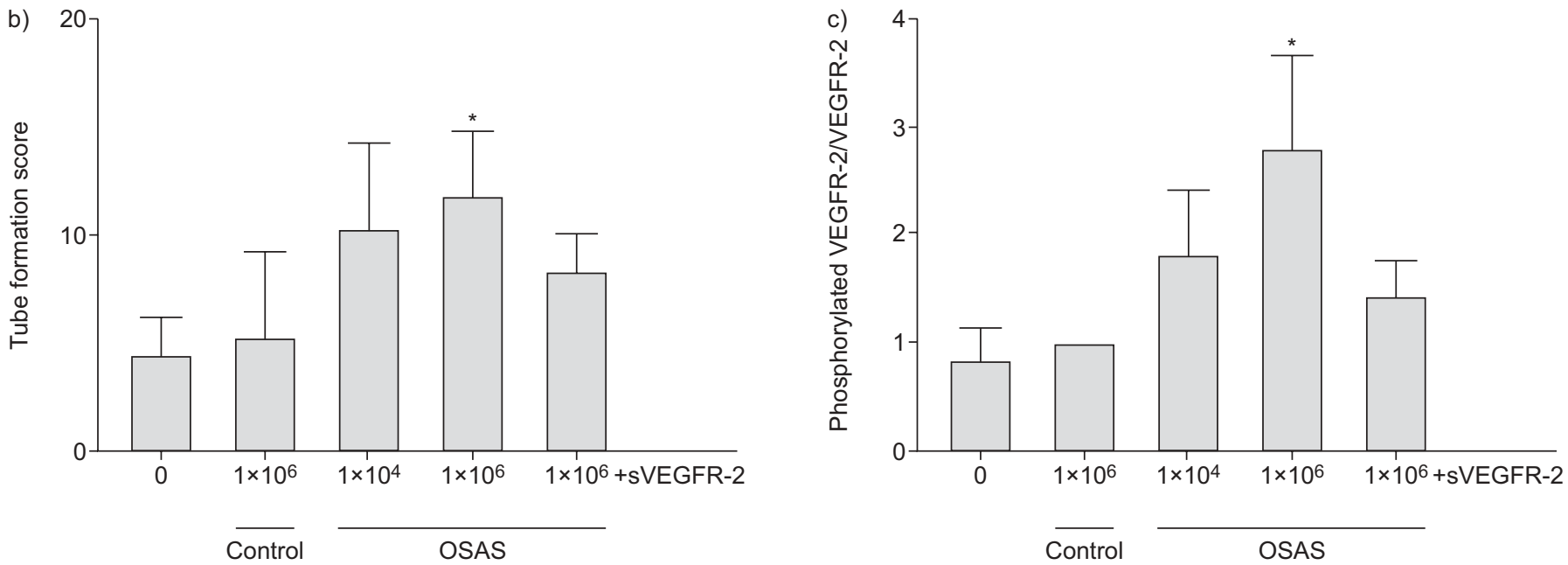

FIGURE 5. Peripheral blood mononuclear cells (PBMCs) from obstructive sleep apnoea syndrome (OSAS) induce tube formation and the phosphorylation on Tyr ${ }^{1214}$ of vascular endothelial growth factor receptor (VEGFR)-2. a) Human aortic endothelial cells (HAECs) were incubated with media alone (-) or co-cultured with $1 \times 10^{6}$ PBMCs from controls or $1 \times 10^{4}$ and $1 \times 10^{6}$ of PBMCs from OSAS patients for 17 days. PBMCs and medium were replaced with PBMCs from another patient in fresh medium every 3 days. At day 17, human umbilical vein endothelial cells (HUVECs) were fixed and submitted to immunostaining with anti-CD31 antibody for HUVECs to detect in vitro angiogenesis, which was assessed by the formation of capillary-like structures of HUVECs co-cultured with PBMCs. Scale bar=100 $\mu \mathrm{m}$. On day 17, HAECs were also fixed and submitted to in-cell western blotting for VEGFR-2 and phosphorylated VEGFR-2 with a Li-Cor Odyssey system (Li-Cor Biosciences, Lincoln, NE, USA). The replacement of PBMCs in the inserts and medium was described in the procedure of the tube formation system. The image represents a 24-well two colour in-cell western blotting with the 800 (green) and 700 (red) channels detecting total VEGFR-2 (as normalisation) and phosphorylated VEGFR-2, respectively. Lanes 1 and 2: endothelial cells (ECs) were cultured only in medium (Lane 1 without anti-VEGFR-2 antibody as a negative control); Lane 3: co-cultured with PBMCs $1 \times 10^{6}$ from control group; Lane 4: co-cultured with PBMCs $1 \times 10^{4}$ from OSAS; Lane 5 and 6: co-cultured with PBMCs $1 \times 10^{6}$ from OSAS (Lane 6: incubation with soluble form of VEGFR-2). b) Ten different fields from the well were randomly selected, and tube formation was evaluated and quantified by the Chalkley counting method. PBMCs from OSAS increased the tube formation of HUVECs. The PBMC-mediated increased tube formation was inhibited by the soluble form of VEGFR-2 (sVEGFR-2). c) In-cell western blotting showed the VEGFR-2 phosphorylation was low in HAECs co-cultured with PBMCs from the control but increased markedly in HAECs co-cultured with PBMCs derived from OSAS in a manner dependent on the number of PBMCs. This increase was suppressed by incubation with soluble VEGFR-2. Phosphorylated VEGFR-2 was normalised by the total VEGFR-2 expression. The fold increase in phosphorylated VEGFR-2 induced by PBMCs was calculated by comparison with the phosphorylated VEGFR-2 on HAECs co-cultured with PBMCs from control. Data are expressed as the mean \pm SEM. * $: p<0.05$ compared with controls.

damage to proteins in human skin fibroblasts [27]. Thus, the study population allowed the evaluation of relationships between ROS and OSAS without the potential influence of confounding factors.

The role of inflammation in the development of atherosclerosis is well established. Once activated by various stimuli (e.g. lowdensity lipoprotein cholesterol, injury, or infection), ROS oxidise lipids, injure cell membranes, create a proinflammatory milieu, and denature the potent vasodilator species nitric oxide [28, 29]. LDL is generally believed to be important in the development of atherosclerosis and its atherogenicity may be due to oxidative modifications, oxLDL [30, 31]. Since LDL is oxidised under the influence of super oxide and incorporated into macrophages, thus forming foam cells, we propose that repetitive hypoxia/ reoxygenation could facilitate free radical production. Also, Ang II was shown to be a potent stimulus for ROS generation [32], which was increased in patients with OSAS when compared with normal control subjects in this study. Our findings are in agreement with MøLLER et al. [33]. They have shown that untreated OSAS is associated with an upregulation of the systemic angiotensin-aldosterone system, and that amelioration of the nocturnal hypoxaemia by long-term $\mathrm{CPAP}$ reduces the blood pressure (BP). Interestingly, this reduction in BP was 
a) PBMC

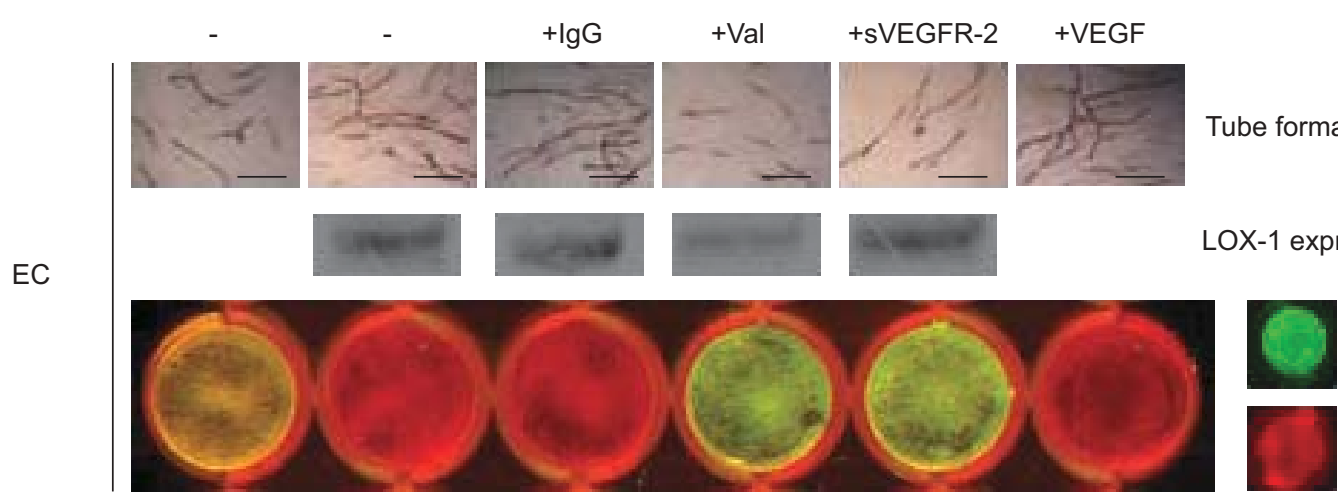

be formation

OX-1 expression
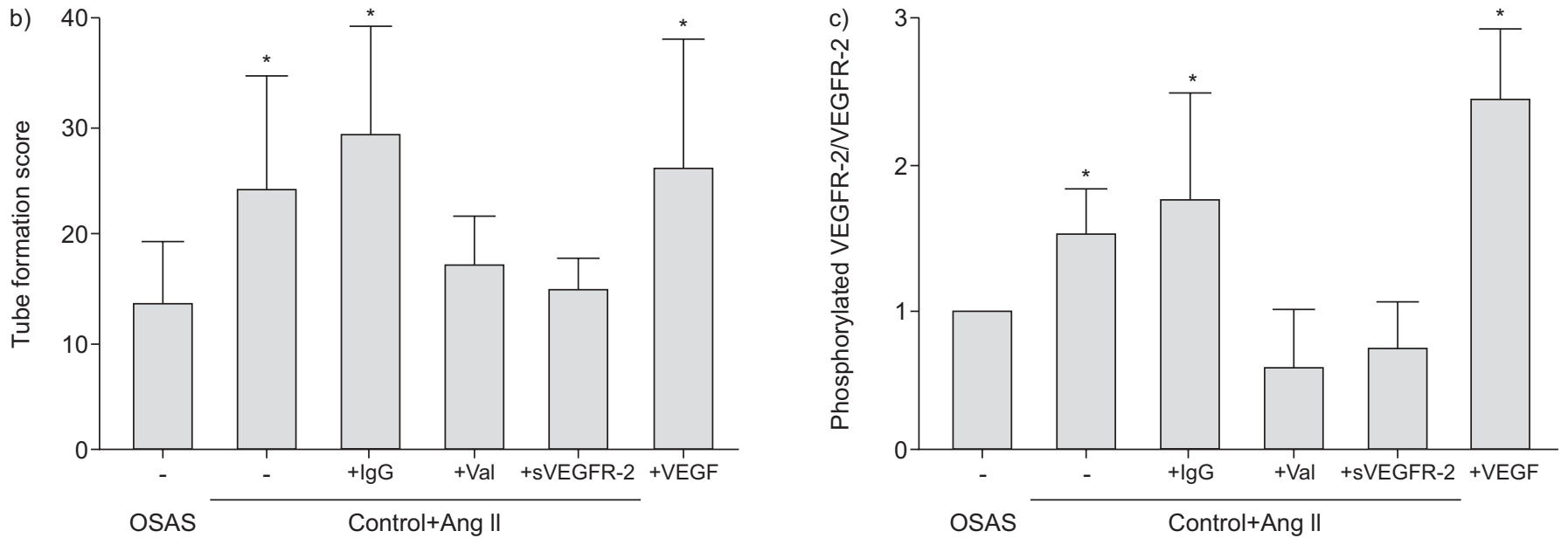

FIGURE 6. Angiotensin (Ang) II and vascular endothelial growth factor (VEGF) from Ang II-stimulated peripheral blood mononuclear cells (PBMCs) induce tube formation, expression of lectin-like oxidised low-density lipoprotein receptor (LOX-1), and the phosphorylation on Tyr ${ }^{1214}$ of VEGF receptor (VEGFR)-2. a) The experimental procedure is described in the legend for figure 5. On day 17, human umbilical vein endothelial cells (HUVECs) were fixed and submitted to immunostaining with anti-CD31 antibody for HUVECs to detect in vitro angiogenesis, which was assessed by the formation of capillary-like structures of HUVECs co-cultured with PBMCs. Scale bar $=100 \mu \mathrm{m}$. On day 17, human aortic endothelial cells (HAECs) were submitted to immunoblotting for LOX-1 expression. HAECs were extracted in sample buffer containing $\beta$-mercaptoethanol. $10 \mu \mathrm{g}$ of proteins were loaded on SDS-PAGE, transferred to nitrocellulose, and analysed by immunoblotting. Note the marked increase in LOX-1 protein in HAECs co-cultured with Ang II-stimulated PBMCs. Incubation of HAECs with valsartan (Val), but not soluble VEGFR (sVEGFR)-2, inhibits Ang II-stimulated PBMCs-induced expression of LOX-1 protein. On day 17, HAECs were also fixed and submitted to in-cell western blotting for VEGFR-2 and phosphorylated VEGFR-2 with a Li-Cor Odyssey system (Li-Cor Biosciences, Lincoln, NE, USA). Replacement of PBMCs in inserts and medium was described in the procedure of the tube formation system. The image represents a 24-well two color in-cell western blotting with the 800 (green) and 700 (red) channels detecting total VEGFR-2 (as normalisation) and phosphorylated VEGFR-2, respectively (bottom). Lane 1: Endothelial cells (ECs) were co-cultured with PBMCs from obstructive sleep apnoea syndrome (OSAS); Lane 2-5: co-cultured with Ang IIstimulated PBMCs from control in the presence of Ang II (Lane 3: pre-treatment with immunoglobulin (Ig) G as a control; Lane 4: pre-treatment with Val; Lane 5: pre-treatment with sVEGFR-2; Lane 6: ECs were cultured only in medium in the presence of VEGF as a positive control. b) Ten different fields from the well were randomly selected, and tube formation was evaluated and quantified by the Chalkley counting method. Ang II-stimulated PBMCs from control increased tube formation of HUVECs. PBMCs-mediated increased tube formation was inhibited by Val and the sVEGFR-2 pre-treatment. c) In-cell western blotting showed that the phosphorylated VEGFR-2 was low in HAECs cocultured with PBMCs from control (fig. 5), but was increased markedly in HAECs co-cultured with Ang II-stimulated PBMCs from control compared with OSAS. This increase was suppressed by incubation with Val and sVEGFR-2. Phosphorylated VEGFR-2 was normalised by the total VEGFR-2 expression. The fold increase in phosphorylation induced by Ang II-stimulated PBMCs or VEGF was calculated by comparison with the phosphorylation in HAECs co-cultured with PBMCs from OSAS. Data are expressed as mean \pm SEM. *: $p<0.05$ compared with OSAS, Val and SVEGFR-2.

strongly correlated with a reduction in the plasma renin and Ang II concentration. Studies by Li et al. [34, 35] have shown that oxLDL and Ang II decrease nitric oxide generation, increase lipid peroxidation and lactate dehydrogenase release in cultured human coronary artery ECs. Thus, it is likely that Ang II and oxLDL are critical factors in atherogenesis. CEPs may play a role in endothelium maintenance, being implicated in both reendothelialisation and neovascularisation. Recent studies show that CEPs are incorporated into new and existing blood vessels in many pathological conditions, including tumour vascularisation and myocardial ischaemia, and that CEPs are elevated and serve as biomarkers for inflammatory diseases such as atherosclerosis [36] and cerebrovascular disease [37]. Indeed, it has been demonstrated that the number of CEPs in the endothelium and in the intima correlated with the magnitude of the presence of hypoxia in patients with chronic obstructive pulmonary disease [38]. 
WERNER et al. [39] have demonstrated that decreased endothelial progenitor cells in patients with coronary artery disease predicts the increased occurrence of cardiovascular events. They have also shown that using mouse model CEPs also contribute to vascular lesion formation by inducing smooth muscle cells proliferation and neointimal formation at sites of vascular injury [40]. Accordingly, CEPs might exert opposite effects on injured vessels. An imbalance between growth factors, facilitated by the disruption of endothelial function and oxidative stress generated by intermittent hypoxaemia, could determine the fate of CEPs. A few groups have reported a decrease or no differences in the number of CEPs between controls and patients with OSAS [1719]. One possible reason for this discrepancy may be due to the differences in studied populations and methods. Their population of controls and patients with OSAS included female subjects. Since several reports have demonstrated that the influence of the menstrual cycle affects the number and the function of CEPs [41, 42], we recruited only male patients and excluded the possibility of the cyclical mobilisation of CEPs. Moreover, since it is also recommended that using CD133, vascular EC marker and CD34 in combination with a viability marker for quantification of EPCs in the blood [43], we used these three antibodies and CD45 to detect CEPs. In our context, VEGF and CEPs are also increased in OSAS patients compared with healthy controls, supporting the concept of a re-endothelialised and neovascularised environment in the OSAS patients. Since there was a CPAP intervention in our study, which has provided further evidence of a link between OSAS and endothelial dysfunction by demonstrating that treatment of apnoea-related hypoxia reduces oxidative stress and angiogenic factors, our present data in vivo supports the notion that oxidative stress, pro-arteriosclerosis and defective reendothelialisation might help to characterise patients with OSAS.

Given this clinical data, in vitro experiments were directed at detecting the interaction of Ang II, oxLDL, PBMCs and ECs. Many studies have shown that LOX-1 facilitates the uptake of oxLDL and mediates several of its biologic effects [44, 45]. KATAOKA et al. [46] have shown that LOX-1 is upregulated in atherosclerotic tissues from humans. In the present study, the expressions of the LOX-1 were upregulated by oxLDL and Ang II (fig. 4). These observations may have important implications with regard to the propagation of EC injury in the presence of Ang II and oxLDL. Another study from our laboratory [10] showed that VEGF expression on PBMCs obtained from patients with OSAS is induced, at least in part, by increased Ang II. VEGF binds to two tyrosine kinase receptors on blood vessel ECs, VEGFR-1/Flt-1 and VEGFR-2/KDR/Flk-1. VEGFR-2 is the most biologically important receptor for VEGF in adults. It regulates EC migration, proliferation, and survival [47]. Following its binding to VEGF, VEGFR-2 dimerises and undergoes autophosphorylation on tyrosine residues within its cytoplasmic portion. We postulated that increased Ang II may enhance VEGF expression and tube formation via the phosphorylation of Tyr ${ }^{1214}$ within VEGFR-2. Indeed, our study demonstrated that Ang II-induced tube formation is dependent on VEGF secreted from PBMCs, and that phosphorylation of $\mathrm{Tyr}^{1214}$ within VEGFR-2 appears during EC tube formation in response to VEGF.

In conclusion, we demonstrated that the levels of Ang II, VEGF, oxLDL and CEPs in peripheral blood in OSAS patients are increased. We also observed an increased angiogenesis when co-cultured with PBMCs obtained from OSAS patients, which was associated with the expression of LOX-1. These findings suggest that recurrent intermittent hypoxaemia may induce an ongoing process of endothelium repair and atherosclerosis. Ang II receptor blocker and nasal CPAP therapy may ultimately represent a potent strategy for clinical therapy of vascular diseases in patients with OSAS.

\section{STATEMENT OF INTEREST}

None declared.

\section{ACKNOWLEDGEMENTS}

The present authors would like to thank the research subjects for participating in these studies and acknowledge the Pulmonary Medicine Research Group staff for their valuable assistance. We would also like to thank N.N. Jarjour, W.W. Busse (Allergy, Pulmonary and Critical Care Section, University of Wisconsin, Madison, WI, USA) and D.P. White (Brigham and Women's Hospital, Harvard Medical School, Boston, MA, USA) for their helpful comments.

\section{REFERENCES}

1 Young T, Palta M, Dempsey J, et al. The occurrence of sleepdisordered breathing among middle-aged adults. $N$ Engl J Med 1993; 328: 1230-1235.

2 Young T, Peppard P. Sleep-disordered breathing and cardiovascular disease: epidemiologic evidence for a relationship. Sleep 2000; 23: S122-S126.

3 Nieto FJ, Young TB, Lind BK, et al. Association of sleep-disordered breathing, sleep apnea, and hypertension in a large communitybased study. Sleep Heart Health Study. JAMA 2000; 283: 1829-1836.

4 Shahar E, Whitney CW, Redline S, et al. Sleep-disordered breathing and cardiovascular disease: cross-sectional results of the Sleep Heart Health Study. Am J Respir Crit Care Med 2001; 163: 19-25.

5 Adair TH, Gay WJ, Montani JP. Growth regulation of the vascular system: evidence for a metabolic hypothesis. Am J Physiol 1990; 259: R393-R404.

6 Leung DW, Cachianes G, Kuang WJ, et al. Vascular endothelial growth factor is a secreted angiogenic mitogen. Science 1989; 246: 1306-1309.

7 Shweiki D, Itin A, Soffer D, et al. Vascular endothelial growth factor induced by hypoxia may mediate hypoxia-initiated angiogenesis. Nature 1992; 359: 843-845.

8 Otani A, Takagi H, Suzuma K, et al. Angiotensin II potentiates vascular endothelial growth factor-induced angiogenic activity in retinal microcapillary endothelial cells. Circ Res 1998; 82: 619-628.

9 Tamarat R, Silvestre JS, Kubis N, et al. Endothelial nitric oxide synthase lies downstream from angiotensin II-induced angiogenesis in ischemic hindlimb. Hypertension 2002; 39: 830-835.

10 Takahashi S, Nakamura Y, Nishijima T, et al. Essential roles of angiotensin II in vascular endothelial growth factor expression in sleep apnea syndrome. Respir Med 2005; 99: 1125-1131.

11 Schulz R, Hummel C, Heinemann S, et al. Serum levels of vascular endothelial growth factor are elevated in patients with obstructive sleep apnea and sever nighttime hypoxia. Am J Respir Crit Care Med 2002; 165: 67-70.

12 Gozal D, Lipton AJ, Jones KL. Circulating vascular endothelial growth factor levels in patients with obstructive sleep apnea. Sleep 2002; 25: 59-65.

13 Imagawa S, Yamaguchi Y, Higuchi M, et al. Levels of vascular endothelial growth factor are elevated in patients with obstructive sleep apnea-hypopnea syndrome. Blood 2001; 98: 1255-1257.

14 Lavie L, Kraiczi H, Hefetz A, et al. Plasma vascular endothelial growth factor in sleep apnea syndrome. Effects of nasal continuous positive air pressure treatment. Am J Respir Crit Care Med 2002; 165: 1624-1628. 
15 Takahashi T, Kalka C, Masuda H, et al. Ischemia- and cytokineinduced mobilization of bone marrow-derived endothelial progenitor cells for neovascularization. Nat Med 1999; 5: 434-438.

16 Asahara $\mathrm{T}$, Masuda $\mathrm{H}$, Takahashi $\mathrm{T}$, et al. Bone marrow origin of endothelial progenitor cells responsible for postnatal vasculogenesis in physiological and pathological neovascularization. Circ Res 1999; 85: 221-228.

17 Martin K, Stanchina M, Kouttab N, et al. Circulating endothelial cells and endothelial progenitor cells in obstructive sleep apnea. Lung 2008; 186: 145-150.

18 de la Peña M, Barceló A, Barbe F, et al. Endothelial function and circulating endothelial progenitor cells in patients with sleep apnea syndrome. Respiration 2008; 76: 28-32.

19 Jelic S, Padeletti M, Kawut SM, et al. Inflammation, oxidative stress, and repair capacity of the vascular endothelium in obstructive sleep apnea. Circulation 2008; 117: 2270-2278.

20 Barceló A, Miralles C, Barbé F, et al. Abnormal lipid peroxidation in patients with sleep apnoea. Eur Respir J 2000; 16: 644-647.

21 Lavie L, Vishnevsky A, Lavie P. Evidence for lipid peroxidation in obstructive sleep apnea. Sleep 2004; 27: 123-128.

22 Wali SO, Bahammam AS, Massaeli $\mathrm{H}$, et al. Susceptibility of LDL to oxidative stress in obstructive sleep apnea. Sleep 1998; 21: 290-296.

23 Svatikova A, Wolk R, Wang $\mathrm{HH}$, et al. Circulating free nitrotyrosine in obstructive sleep apnea. Am J Physiol Regul Integr Comp Physiol 2004; 287: R284-R287.

24 Dyugovskaya, Lavie P, Lavie L. Increased adhesion molecules expression and production of reactive oxygen species in leukocytes of sleep apnea patients. Am J Respir Crit Care Med 2002; 165: 934-939.

25 Nakamura Y, Esnault S, Maeda T, et al. Ets-1 regulates TNF- $\alpha$ induced matrix metalloproteinase- 9 and tenascin expression in primary bronchial fibroblasts. J Immunol 2004; 172: 1945-1952.

26 Kortum RL, Lewis RE. The molecular scaffold KSR1 regulates the proliferative and oncogenic potential of cells. Mol Cell Biol 2004; 24: 4407-4416.

27 Hoerter JD, Ward CS, Bale KD, et al. Effect of UVA fluence rate on indicators of oxidative stress in human dermal fibroblasts. Int $J$ Biol Sci 2008; 4: 63-70.

28 Landmesser U, Hornig B, Drexler H. Endothelial function: a critical determinant in atherosclerosis? Circulation 2004; 109: II27-II33.

29 McCord JM. Oxygen-derived radicals: a link between reperfusion injury and inflammation. Fed Proc 1987; 46: 2402-2406.

30 Witztum JL, Steinberg D. Role of oxidized low density lipoprotein in atherogenesis. J Clin Invest 1991; 88: 1785-1792.

31 Steinberg D, Parthasarathy S, Carew TE, et al. Beyond cholesterol. Modifications of low-density lipoprotein that increase its atherogenicity. N Engl J Med 1989; 320: 915-924.
32 Ushio-Fukai M. Redox signaling in angiogenesis: role of NADPH oxidase. Cardiovasc Res 2006; 71: 226-235.

33 Møller DS, Lind P, Strunge B, et al. Abnormal vasoactive hormones and 24-hour blood pressure in obstructive sleep apnea. Am J Hypertens 2003; 16: 274-280.

34 Li D, Yang B, Philips MI, et al. Proapoptotic effects of ANG II in human coronary artery endothelial cells: role of AT1 receptor and PKC activation. Am J Physiol 1999; 276: H786-H792.

35 Li D, Yang B, Mehta JL. Ox-LDL induces apoptosis in human coronary artery endothelial cells: role of PKC, PTK, bcl-2, and Fas. Am J Physiol 1998; 275: H568-H576.

36 Rauscher FM, Goldschmidt-Clermont PJ, Davis BH, et al. Aging, progenitor cell exhaustion, and atherosclerosis. Circulation 2003; 108: 457-463.

37 Ghani U, Shuaib A, Salam A, et al. Endothelial progenitor cells during cerebrovascular disease. Stroke 2005; 36: 151-153.

38 Peinado VI, Ramírez J, Roca J, et al. Identification of vascular progenitor cells in pulmonary arteries of patients with chronic obstructive pulmonary disease. Am J Respir Cell Mol Biol 2006; 34: 257-263.

39 Werner N, Kosiol S, Shiegl T, et al. Circulating endothelial progenitor cells and cardiovascular outcomes. $N$ Engl J Med 2005; 353: 999-1007.

40 Werner N, Priller J, Laufs $\mathrm{U}$, et al. Bone marrow-derived progenitor cells modulate vascular reendothelialization and neointimal formation. Effect of 3-hydroxy-3-methylglutaryl coenzyme a reductase inhibition. Arterioscler Thromb Vasc Biol 2002; 22: $1567-1572$.

41 Fadini GP, de Kreutzenberg S, Albiero M, et al. Gender differences in endothelial progenitor cells and cardiovascular risk profile: the role of female estrogens. Arterioscler Thromb Vasc Biol 2008; 28: 997-1004.

42 Robb AO, Mills NL, Smith IB, et al. Influence of menstrual cycle on circulating endothelial progenitor cells. Hum Reprod 2009; 24 615-625.

43 Distler JH, Allanore Y, Avouac J, et al. EULAR Scleroderma trials and research group statement and recommendations on endothelial precursor cells. Ann Rheum Dis 2009; 68: 163-168.

44 Sawamura T, Kume N, Aoyama T, et al. An endothelial receptor for oxidized low-density lipoprotein. Nature 1997; 386: 73-77.

45 Mehta JL, Li DY. Identification and autoregulation of receptor for OX-LDL in cultured human coronary artery endothelial cells. Biochem Biophys Res Commun 1998; 248: 511-514.

46 Kataoka H, Kume N, Miyamoto S, et al. Expression of lectinlike oxidized low-density lipoprotein receptor-1 in human atherosclerotic lesions. Circulation 1999; 99: 3110-3117.

47 Rousseau S, Houle F, Huot J. Integrating the VEGF signals leading to actin-based motility in vascular endothelial cells. Trends Cardiovasc Med 2000; 10: 321-327. 